\title{
Power spectrum optimization for interference mitigation via iterative function evaluation
}

\author{
Hayssam Dahrouj ${ }^{1}$, Wei Yu $^{{ }^{*}}$ and Taiwen Tang ${ }^{2}$
}

\begin{abstract}
Power spectrum optimization is a well-known difficult nonconvex optimization problem for which only local optimality can typically be assured. This paper unifies several classes of local optimization methods and proposes efficient and new methods for power spectrum optimization by observing that methods for reaching the local optimal points can often be expressed in the form of an iterative function evaluation. This proposed new approach is based on the fact that the gradient of the objective function is zero at a local optimum, and that different manipulations of the optimality condition can then lead to different power update equations. As a practical application, this paper examines the benefit of dynamic power spectrum optimization for interference mitigation in a wireless backhaul network in which remote radio units are deployed to serve mobile users in areas with high data traffic demand. The remote radio units, called remote terminals (RT), are connected to access nodes (AN) via orthogonal frequency division multiple access (OFDMA) over a fixed bandwidth with one RT active in each frequency tone. The system performance is thus limited by internode interference solely, and no intranode interference. This paper shows that iterative function evaluation based methods provide a significant improvement in the overall network throughput in this setting as compared to a conventional network with fixed transmit power spectrum. The proposed methods have computationally fast convergence and can be implemented in a distributed fashion assuming reasonable amount of internode information exchange. Further, some of the proposed methods can be implemented asynchronously at each AN, which makes them amenable to practical utilization.
\end{abstract}

\section{Introduction}

Interference is a major bottleneck in wireless network design. Developing and optimizing advanced, yet practical, interference mitigation techniques is particularly important nowadays, due to the rapid pace of growth of wireless networks with enormous data usage, and the scarcity of the available radio resources, e.g. bandwidth and transmit power. Dynamic power spectrum optimization is an important class of interference mitigation methods that seek to increase the network capacity and reliability via power control. The present paper aims to develop novel, feasible, practical methods for power spectrum optimization.

Dynamic power spectrum optimization is especially important to distributed antenna systems (DAS) where the base-station transmit capability is enhanced by adding

\footnotetext{
*Correspondence: weiyu@comm.utoronto.ca

1 Electrical and Computer Engineering Department, University of Toronto,

Toronto, Canada

Full list of author information is available at the end of the article
}

multiple remote radio units. This paper focuses on a wireless cellular network comprising multiple remote terminals (RT), each covering a relatively small area, called a picocell, as a means to increase the network capacity for areas with dense data traffic. The mobile users within each picocell are served by the picocell base-station, which are collocated with the RTs. The RTs are then connected to access nodes (ANs) via wireless backhaul links, which are meant to replace the expensive optical fiber links. This paper assumes that the ANs can be coordinated in transmission strategies and radio resource management when transmitting to different RTs. From a design perspective, the interest of this paper is to mitigate the internode interference, thereby maximizing the aggregate data capacity to the RTs via practical power spectrum optimization methods.

This paper adopts a network utility maximization framework and focuses on the weighted rate-sum maximization problem, which is known to be a difficult nonconvex problem. Consequently, most numerical methods focus on finding its local optimum only. The main point

\section{是 Springer}

(c) 2012 Dahrouj et al. licensee Springer. This is an Open Access article distributed under the terms of the Creative Commons Attribution License (http://creativecommons.org/licenses/by/2.0), which permits unrestricted use, distribution, and reproduction in any medium, provided the original work is properly cited. 
of this paper is that under power spectral density (PSD) constraints, the local optimization of weighted rate-sum in an interference environment can often be performed efficiently via a class of methods based on iterative function evaluation. This proposed new approach is based on the observation that local optima of an optimization problem occur where the gradient of the objective function is zero, and that different manipulations of the gradient function can lead to different numerical power update algorithms. The power update equations obtained this way take the form of an iterative function evaluation. The performance and convergence properties of iterative function evaluation methods (IFEM) for power spectrum optimization are the focus of this paper.

While power spectrum optimization methods have been widely proposed in the literature, the practical implementation of power adaptation in wireless applications is often challenging because wireless channels can vary rapidly over time, and because wireless transmitters are often located in geographically separated locations. Thus, computational complexity and speed of convergence are key considerations for power adaptation in wireless applications; further, distributed and asynchronous implementation are often desired. The main advantage of the iterative function evaluation based power adaptation methods proposed in this paper is that they typically have a low computational complexity, while showing a significant performance gain as compared to the conventional maximum power transmission policy. Further, these methods lend themselves to distributed implementation with reasonable amount of internode information exchange. Some of the methods can also be implemented asynchronously, which makes them amenable to practical utilization.

One of the objectives of this paper is to quantify the benefit of power spectrum optimization in a practical wireless backhaul deployment. This paper envisions a setting that makes use of channel measurements done on a per-frequency-tone basis for every AN-RT pair. The measurements are subsequently provided either to a central server for further centralized processing, or to each of the several ANs for distributed processing. To minimize the overhead of such exchange of information, the channel measurements can alternatively take the form of frequency domain average channel gains. The performance of the proposed methods with such average channel gains is also quantified in this paper. The power spectrum optimization methods proposed in this paper are of particular interest in a fixed deployment scenario such as the wireless backhaul network, where the channels are relatively slow varying. Further, the measurements can be done periodically, thus allowing the adaptation of radio resource allocations with the dynamically changing environment.

\section{Related work}

The main challenge in dynamic power spectrum management is that of finding computationally efficient methods to allocate powers to different transmitters across the different frequency tones in order to maximize some overall system wide utility. This power spectrum optimization problem is especially well studied in the literature for the digital subscriber line (DSL) system [1-5]. In particular, the works $[4,5]$ make use of concept known as interference price, which is related to the methods proposed in this paper. Interference price quantifies the effect of interference between the multiple transmitter-receiver pairs. The use of interference pricing for power spectrum adaptation in fact originated from the wireless literature [6-9], where various power control algorithms are studied.

In the wireless application, power control methods need to be incorporated with scheduling. Joint power control and scheduling has been investigated in the literature for both single-cell $[10,11]$ and multicell systems [12-15]. The overall problem setup of this paper is closely related to that in $[14,15]$, where power spectrum adaptation is jointly performed with proportionally fair scheduling. The contribution of this paper focuses on power control.

The main focus on this paper is on the efficiencycomplexity tradeoff of different power control algorithms. The weighted sum-rate maximization problem studied in this paper has a well-known and difficult nonconvex structure induced by the coupling between the power terms. The solutions referenced above (and the new algorithms proposed in this paper), therefore, only reach local optimal points. Finding the global optimum of the spectrum management problem is typically much more difficult. Toward this end, an algorithm called MAPEL is recently proposed in [16] based on monotonic optimization and fractional programming, and it is guaranteed to converge to the global optimal solution. The computational complexity of implementing MAPEL can however be high. Further, it requires centralized processing; its convergence speed also remains an open problem.

This paper proposes novel, low-complexity, but local algorithms to solve the weighted sum-rate maximization problem. The proposed methods are based on the manipulations of the gradient of the objective function. Although they only guarantee local optimality, as the simulations results show, they already bring in significant improvement as compared to conventional systems with the maximum power transmission strategy. The main advantages of this new class of algorithms are efficiency and potential for distributed and asynchronous implementation. In particular, they do not require step size choices, as traditional subgradient or Newton's methods in optimization do. 


\section{System model and problem formulation System model}

Consider a wireless multicell network with $L$ cells with one $\mathrm{AN}$ and $K$ RTs per cell, and with single antennas at both the ANs and RTs. The RTs belonging to each AN are separated from each other using orthogonal frequency division multiple access (OFDMA) with $N$ tones over a fixed bandwidth, where only one RT is active at each frequency tone. This paper focuses on the downlink backhaul transmission from the ANs to the RTs, and aims to use power management methods to alleviate inter-AN interference. In particular, the $l$ th $\mathrm{AN}$ may allocate its power $P_{l}^{n}$ at each tone $n \in\{1, \cdots, N\}$, depending on the scheduling assignment of RTs and the channel gains between the AN-RT pairs. Let $k=f(l, n)$ and $k^{\prime}=f(j, n)$ be the scheduled RTs at the $n$th tone for the $l$ th AN and $j$ th AN respectively. The received signal at the $k$ th $\mathrm{RT}$ in the $n$th frequency tone is a summation of the intended signal and the internode interference:

$$
y_{l}^{n}=h_{l l k}^{n} x_{l}^{n}+\sum_{j \neq l} h_{j l k}^{n} x_{j}^{n}+z_{l}^{n}
$$

where $x_{l}^{n}$ is a complex scalar denoting the information signal intended for the $k$ th RT served by the $l$ th AN, $x_{j}^{n}$ is a complex scalar denoting the information signal intended for the $k^{\prime}$ th RT served by the $j$ th $\mathrm{AN}, h_{j l k}^{n} \in \mathbb{C}$ is the channel from the $j$ th AN to the $k$ th RT in the $l$ th cell, and $z_{l}^{n}$ is the additive white Gaussian noise with variance $\sigma^{2} / 2$ on each of its real and imaginary components. For simplicity, all the ANs and RTs are assumed to be equipped with one antenna each. Figure 1 illustrates the system model for seven ANs and four RTs per AN.

\section{Problem formulation}

This paper focuses on solving the following weighted sumrate optimization problem:

$$
\begin{array}{ll}
\max & \sum_{l, k} w_{l k} r_{l k}^{n} \\
\text { s.t. } & 0 \leq P_{l}^{n} \leq S^{\text {max }} \\
& k=f(l, n)
\end{array}
$$

where

$$
r_{l k}^{n}=\log \left(1+\frac{P_{l}^{n}\left|h_{l l k}^{n}\right|^{2}}{\Gamma\left(\sigma^{2}+\sum_{j \neq l} P_{j}^{n}\left|h_{j l k}^{n}\right|^{2}\right)}\right)
$$

is the instantaneous rate of the scheduled $k$ th $\mathrm{RT}$ for the $l$ th $\mathrm{AN}$ at the $n$th tone, $S^{\max }$ is the maximum power constraint imposed on each $\mathrm{AN}$ at each tone, and where the maximization is over the scheduling assignment $k=$ $f(l, n)$, and the power spectral density levels $P_{l}^{n}$. The $\Gamma$ is the signal-to-noise ratio (SNR) gap. The weights $w_{l k}$ here come typically from upper layer considerations that account for queue length, traffic demand and user priorities.

The problem (2) is a mixed discrete-continuous optimization problem, the global optimal solution of which may require an exhaustive search with exponential complexity. This paper, however, focuses on the power allocation step and assumes a fixed scheduling policy. For a fixed schedule, the weighted rate-sum maximization problem

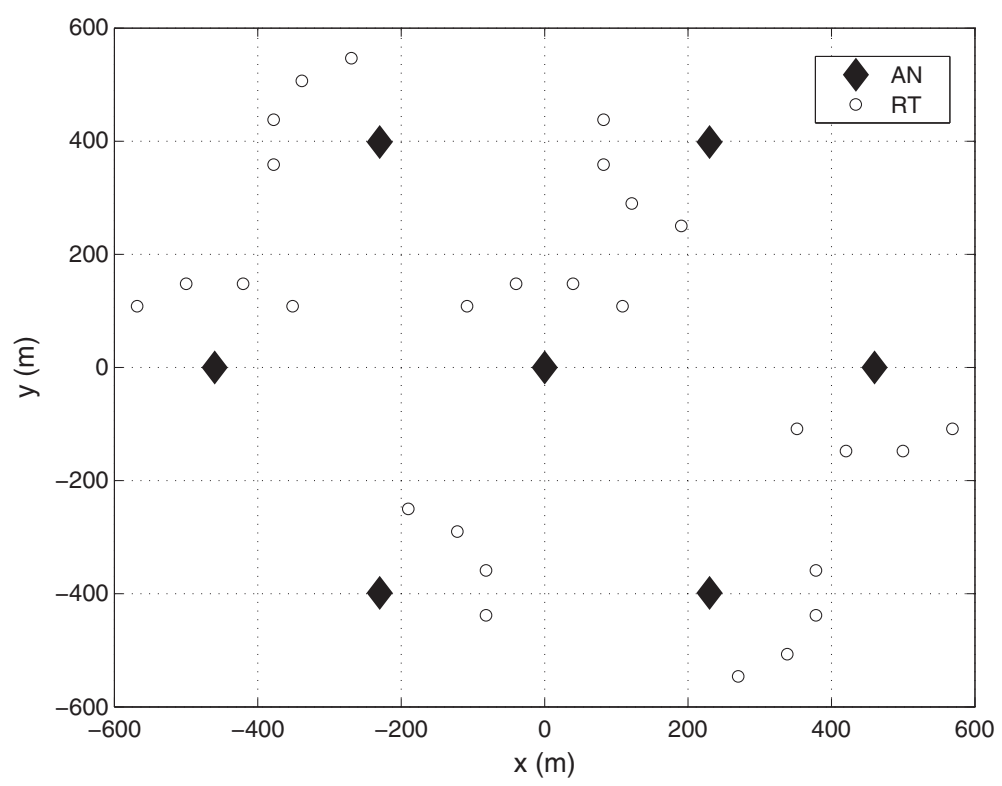

Figure 1 A distributed antenna system with seven 7 ANs and 4 RTs per AN. 
reduces on a per-tone basis. For each frequency tone $n$, the optimization problem is now

$$
\begin{aligned}
& \max \sum_{l} w_{l k} r_{l k}^{n} \\
& \text { s.t. } \quad 0 \leq P_{l}^{n} \leq S^{\max }
\end{aligned}
$$

where the maximization is over the set of powers $P_{l}^{n}$.

Note that, in general, problem formulation (2) can be solved via an iterative scheduling and power control policy similar to that in [13-15], in which the scheduling is done assuming fixed power, and power optimization is done assuming a fixed schedule. The iterative algorithm is based on the fact that for fixed power $P_{l}^{n} \forall(l, n)$, the interference produced by a AN $j$ to an RT $k$, scheduled for the $l$ th AN at the $n$th tone $(l \neq j)$, is a function of the set of power levels only, and is independent of the scheduling choice of RTs at the $j$ th AN. For fixed power $P_{l}^{n} \forall(l, n)$, the scheduling can therefore be done on per-AN basis without affecting the inter-AN interference levels. More specifically, the RT scheduled for the $l$ th AN at the $n$th tone is the one that satisfies the following equation:

$$
k=f(l, n)=\operatorname{argmax}_{\xi} w_{l \xi} r_{l \xi}^{n}
$$

where

$$
r_{l \xi}^{n}=\log \left(1+\frac{P_{l}^{n}\left|h_{l l \xi}^{n}\right|^{2}}{\Gamma\left(\sigma^{2}+\sum_{j \neq l} P_{j}^{n}\left|h_{j l \xi}^{n}\right|^{2}\right)}\right)
$$

is the instantaneous rate of the scheduled $\xi$ th RT for the $l$ th $\mathrm{AN}$ at the $n$th tone. The rest of the paper examines practical numerical methods to solve (4), i.e. under fixed scheduling.

This paper chooses to use a power spectrum density (PSD) constraint which particularly simplifies the numerical optimization procedure. Although the numerical solution of this paper can also adapted to the case where total power constraints are also present, one could argue that PSD constraints occur more naturally in practical settings, and is therefore adopted in this paper.

\section{Power spectrum optimization}

The weighted sum-rate maximization problem (4) is a nonconvex optimization problem, whose global optimal solution is not easy to find. Like many previous approaches, this paper also aims at local optimal solutions, but with a focus on reducing computational complexity. Traditional optimization approaches for solving a nonlinear optimization problem include gradient projection or Newton's method. In contrast, this paper proposes a new class of strategies based on an iterative function evaluation approach. These novel methods, described below, are simple to implement, fast in convergence, and similar in performance to traditional full-blown Newton's method. In addition, they have the advantages of being amenable to distributed and asynchronously implementation, and are free of step size choices.

The proposed methods all result from different manipulations of the gradient of the objective function. Below, we first present two "full" methods, called IFEM-1 and IFEM2 . We then present simplified versions that offer further reduced computational complexity. Since the objective function in (4) is not necessarily concave, the approach used in deriving IFEM-1 and IFEM-2 only looks for stationary points to the problem. Nevertheless, the proposed solutions are shown to provide a significant performance improvement as compared to conventional systems.

\section{Iterative function evaluation methods \\ IFEM-1}

The objective function of the problem (4), which is optimized over the set of power on a tone-by-tone basis, can be written as:

$$
R\left(P_{1}^{n}, P_{2}^{n}, \cdots, P_{L}^{n}\right)=\sum_{l} w_{l k} \log \left(1+\frac{P_{l}^{n}\left|h_{l l k}^{n}\right|^{2}}{\Gamma\left(\sigma^{2}+\sum_{j \neq l} l_{j}^{n}\left|h_{j l k}^{n}\right|^{2}\right)}\right)
$$

We begin by taking the derivative of the objective function $R$ with respect to $P_{l}^{n}$ :

$$
\begin{aligned}
\frac{\partial R}{\partial P_{l}^{n}}= & w_{l k} \frac{\partial r_{l k}^{n}}{\partial P_{l}^{n}}+\sum_{j \neq l} w_{j k^{\prime}} \frac{\partial r_{j k^{\prime}}^{n}}{\partial P_{l}^{n}} \\
= & \frac{w_{l k}}{P_{l}^{n}}\left(\frac{\operatorname{SINR}_{l}^{n}}{1+\operatorname{SINR}_{l}^{n}}\right) \\
& -\sum_{j \neq l} w_{j k^{\prime}} \frac{\left|h_{l j k^{\prime}}^{n}\right|^{2}}{\sigma^{2}+\sum_{i \neq j} P_{i}^{n}\left|h_{i j k^{\prime}}^{n}\right|^{2}} \frac{\operatorname{SINR}_{j}^{n}}{1+\operatorname{SINR}_{j}^{n}},
\end{aligned}
$$

where $\operatorname{SINR}_{j}^{n}$ is defined as:

$$
\operatorname{SINR}_{j}^{n}=\frac{P_{j}^{n}\left|h_{j j k^{\prime}}^{n}\right|^{2}}{\Gamma\left(\sigma^{2}+\sum_{i \neq j} P_{i}^{n}\left|h_{i j k^{\prime}}^{n}\right|^{2}\right)},
$$

and $k=f(l, n)$ and $k^{\prime}=f(j, n)$ are the scheduled RTs of the $l$ th AN and the $j$ th AN respectively at the $n$th tone. A local optimal solution must be such that the above gradient is zero. The key idea of this paper is that by setting the above gradient to zero and by manipulating the optimality condition, one can obtain algorithms for optimizing power. For example, one possible manipulation is:

$$
P_{l}^{n}=\frac{w_{l k} \frac{\operatorname{SINR}_{l}^{n}}{1+\operatorname{SINR}_{l}^{n}}}{\sum_{j \neq l} w_{j k^{\prime}} \frac{\left|h_{l j k^{\prime}}^{n}\right|^{2}}{\sigma^{2}+\sum_{i \neq j} P_{i}^{n}\left|h_{i j k^{\prime}}^{n}\right|^{2}} \frac{\operatorname{SINR}_{j}^{n}}{1+\operatorname{SINR}_{j}^{n}}} .
$$

The point is that it is possible to interpret the above condition as an iterative algorithm for computing the optimal $\left\{P_{l}^{n}\right\}_{l=1}^{L}$. In other words, given the current set of power allocation, one can compute the terms on the right-handside of the above equation using the current power, then use the result to update the new power allocation. This 
step is a simple function evaluation, which can be done iteratively. Hence this method is named IFEM-1.

To account for the PSD constraints, an additional projection on the constraint set $[.]_{0}^{S^{\max }}$ can be taken. More formally, in each iteration of the algorithm IFEM-1, the power level of every AN at every tone, $P_{l}^{n}$, is updated from step $t$ to $t+1$ according to the following:

$$
P_{l}^{n}(t+1)=\left[\frac{w_{l k} \frac{\operatorname{SINR}_{l}^{n}(t)}{1+\operatorname{SINR}_{l}^{n}(t)}}{\sum_{j \neq l} \tau_{j l}^{n}(t)}\right]_{0}^{S^{\max }},
$$

where

$$
\tau_{j l}^{n}(t)=\frac{w_{j k^{\prime}}\left|h_{i j k^{\prime}}^{n}\right|^{2}}{\sigma^{2}+\sum_{i \neq j} P_{i}^{n}(t)\left|h_{i j k^{\prime}}^{n}\right|^{2}} \frac{\operatorname{SINR}_{j}^{n}(t)}{1+\operatorname{SINR}_{j}^{n}(t)} .
$$

Note that if (and when) the iterative process converges, it must converge to a point that satisfies the stationarity condition. Thus, the convergence point must be a local stationary point, (and often observed to be a local optimum in practice). This is obvious to see when the converged point is in the interior of the constrained set, but is also true when the converged point is on the boundary. In this latter case, it can be shown that a suitable set of Lagrangian multipliers can be found so that the converged point satisfies the KKT conditions of the optimization problem. A detailed proof can be found in [5]. Note that the $\tau_{j l}^{n}$ term defined above is an interference pricing term. It is a function of the channel gain $\left|h_{l j k^{\prime}}^{n}\right|^{2}$ and indicates the impact of the interference from the $l$ th $\mathrm{AN}$ to the scheduled RT of the $j$ th $\mathrm{AN}$ at the $n$th tone. As the interference price $\tau_{j l}^{n}$ increases, the $l$ th AN tends to decrease its power $P_{l}^{n}$. In a distributed implementation, these pricing terms are exactly the information that needs to be exchanged among the ANs.

\section{IFEM-2}

Interestingly, the above derivation is not the only way to derive an iterative algorithm for optimizing power. In fact, different manipulations of the optimality condition can lead to different algorithms. For example, starting again by setting the gradient (7) to zero:

$$
\begin{aligned}
\frac{\partial R}{\partial P_{l}^{n}}= & \frac{w_{l k}}{P_{l}^{n}}\left(1+\frac{1}{\operatorname{SINR}_{l}^{n}}\right)^{-1} \\
& -\sum_{j \neq l} w_{j k^{\prime}} \frac{\left|h_{l j k^{\prime}}^{n}\right|^{2}}{\sigma^{2}+\sum_{i \neq j} P_{i}^{n}\left|h_{i j k^{\prime}}^{n}\right|^{2}} \frac{\operatorname{SINR}_{j}^{n}}{1+\operatorname{SINR}_{j}^{n}}=0,
\end{aligned}
$$

we can write

$$
P_{l}^{n}\left(1+\frac{1}{\operatorname{SINR}_{l}^{n}}\right)=\frac{w_{l k}}{\sum_{j \neq l} w_{j k^{\prime}} \frac{\left|h_{l j k^{\prime}}^{n}\right|^{2}}{\sigma^{2}+\sum_{i \neq j} P_{i}^{n}\left|h_{i j k^{\prime}}^{n}\right|^{2}} \frac{\operatorname{SINR}_{j}^{n}}{1+\operatorname{SINR}_{j}^{n}}} .
$$

Thus,

$$
P_{l}^{n}=\frac{w_{l k}}{\sum_{j \neq l} w_{j k^{\prime}} \frac{\left|h_{l j k^{\prime}}^{n}\right|^{2}}{\sigma^{2}+\sum_{i \neq j} P_{i}^{n}\left|h_{i j k^{\prime}}^{n}\right|^{2}} \frac{\operatorname{SINR}_{j}^{n}}{1+\operatorname{SINR}_{j}^{n}}}-\frac{P_{l}^{n}}{\operatorname{SINR}_{l}^{n}} .
$$

Again, we can interpret the above equation as an iterative algorithm. As before, one can compute the terms on the right-hand side, and update the new power allocation according to (12). We name the resulting new algorithm IFEM-2. More formally, in IFEM-2 the power level of every AN at every tone, $P_{l}^{n}$, is updated from step $t$ to $t+1$ according to

$$
P_{l}^{n}(t+1)=\left[\frac{w_{l k}}{\sum_{j \neq l} \tau_{j l}^{n}(t)}-\frac{P_{l}^{n}(t)}{\operatorname{SINR}_{l}^{n}(t)}\right]_{0}^{S^{\max }}
$$

where the power constraint is again taken into account though the projection step.

\section{High-SINR IFEM}

The above manipulations of the optimality condition are by no means the only ways to derive a fixed-point equation for power optimization. Numerous possibilities exist, but the above two are the most sensible. These IFEM algorithms, if converging, likely converge to a local optimum solution of the original problem. The key differentiating factor is therefore their convergence properties.

Unfortunately, the convergence of the IFEM-1 and IFEM-2 is not easy to establish in full generality, due to the tight coupling of the interference terms. But, there are specific simplifications of the algorithms for which convergence proofs are possible. For example, consider a high SINR scenario. In this case, both IFEM-1 and IFEM-2 reduce to the same update equation shown below:

$$
P_{l}^{n}(t+1)=\left[\frac{w_{l k}}{\sum_{j \neq l} w_{j k^{\prime}} \frac{\left|h_{i j k^{\prime}}^{n}\right|^{2}}{\sigma^{2}+\sum_{i \neq j} P_{i}^{n}(t)\left|h_{i j k^{\prime}}^{n}\right|^{2}}}\right]_{0}^{S^{\max }}
$$

We call the resulting algorithm high-SINR IFEM (HSIFEM). As shown later in the paper, the above update algorithm can be shown to always converge, but HSIFEM is no longer guaranteed to converge to a local optimal point due to the omission of SINR terms.

\section{$\theta$-IFEM- 1 and $\theta$-IFEM-2}

Thus far, we have on one hand IFEM-1 and IFEM-2 algorithms, for which convergence is not easy to establish, but if they do converge, they converge to a local optimum. On the other hand, we have HSIFEM which is guaranteed to converge, but not necessarily to a local optimum. To make further progress, we may consider compromises along the following directions.

For IFEM-1, we can consider a method that replaces the per-iteration SINR's in the IFEM-1 update equation 
with fixed values of SINR's calculated under the initial maximum power transmission strategy. Although this also does not guarantee local optimality, it has the advantage that its convergence is easy to establish, and it is a better approximation of the IFEM-1 algorithm than the highSNR approximation. This method, called $\theta$-IFEM- 1 in this paper, finds the power level $P_{l}^{n}$ according to the following update equation:

$$
P_{l}^{n}(t+1)=\left[\frac{w_{l k}}{\sum_{j \neq l} w_{j k^{\prime}} \frac{\left|h_{l j j^{\prime}}^{n}\right|^{2}}{\sigma^{2}+\sum_{i \neq j} P_{i}^{n}(t)\left|h_{i j k^{\prime}}^{n}\right|^{2}} \theta_{j l}^{n}}\right]_{0}^{S^{\max }}
$$

where

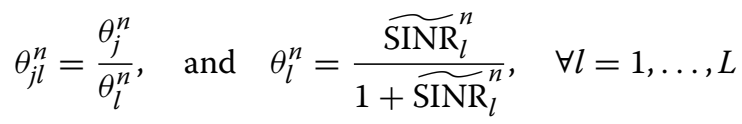

and where $\widetilde{\operatorname{SINR}}_{l}^{n}$ is the fixed SINR calculated from the maximum power transmission strategy, i.e.

$$
{\widetilde{\operatorname{SINR}_{l}}}_{l}^{n}=\frac{S^{\max }\left|h_{l k}^{n}\right|^{2}}{\Gamma\left(\sigma^{2}+\sum_{j \neq l} S^{\max }\left|h_{j l k}^{n}\right|^{2}\right)}
$$

Note that HSIFEM is simply $\theta$-IFEM- 1 with $\theta_{j l}^{n}=1$.

A similar method can be applied to IFEM-2, i.e. replacing the per-iteration SINRs in the IFEM-2 update equation with the values of SINR's calculated under the initial maximum power transmission strategy. However, it turns out that this method does not necessarily converge. But for the sake of completeness, we write down the $\theta$-IFEM-2 algorithm, which updates the power level $P_{l}^{n}$ according to the following equation:

$$
P_{l}^{n}(t+1)=\left[\frac{w_{l k}}{\sum_{j \neq l} \frac{\left.w_{j k^{\prime}}||_{l j k^{\prime}}^{n}\right|^{2}+\sum_{i \neq j} P_{i}^{n}(t)\left|h_{l j k^{\prime}}^{n}\right|^{\prime}}{\sigma_{j}^{n}}}-\frac{P_{l}^{n}(t)}{\frac{\theta_{l}^{n}}{1-\theta_{l}^{n}}}\right]_{0}^{S^{\max }}
$$

\section{Convergence analysis}

As mentioned earlier, the convergence of IFEM-1 and IFEM-2 is difficult to establish in full generality, but $\theta$ IFEM-1 and HSIFEM algorithms always converge. This section establishes this fact using the theory of standard functions.

Proposition 1. Starting from any initial feasible $P_{l}^{n}(0), \theta$ IFEM-1 and HSIFEM algorithms respectively converge to unique fixed points. Furthermore, the convergence is still guaranteed under a totally asynchronous model.

Proof. The proof is based on Corollary 1 in [17], as both update equations of $\theta$-IFEM- 1 and HSIFEM, written as
$P_{l}^{n}(t+1)=\left[g_{l}\left(\Psi^{n}(t)\right)\right]_{0}^{S^{\max }}$, satisfy the following standard function properties:

1. If $P_{l}^{n} \geq 0 \forall l, n$, then $g_{l}\left(\Psi^{n}\right)>0$.

2. If $P_{l}^{n} \geq P^{\prime n}{ }_{l}^{\prime} \forall l, n$, then $g_{l}\left(\Psi^{n}\right) \geq g_{l}\left(\Psi^{\prime n}\right)$.

3. For $\rho>1$, we have $\rho g_{l}\left(\Psi^{n}\right)>g_{l}\left(\rho \Psi^{\mathbf{n}}\right) \forall l, n$.

where the variables $P_{l}^{n}, l=1, \cdots, L$, are stacked into one vector $\Psi^{n}$. The convergence to the unique fixed point and the asynchronous convergence follow as a consequence.

We show here how briefly the above conditions hold for $\theta$-IFEM-1. Similar steps apply for HSIFEM. Consider the update equation of $\theta$-IFEM-1:

$$
P_{l}^{n}=\left[\frac{w_{l k}}{\sum_{j \neq l} w_{j k^{\prime}} \frac{\left|h_{l i k^{\prime}}^{n}\right|^{2}}{\sigma^{2}+\sum_{i \neq j} P_{i}^{n}\left|h_{i j k^{\prime}}^{n}\right|^{2}} \theta_{j l}^{n}}\right]_{0}^{S^{\max }}
$$

Define the function $f_{l}($.$) as:$

$$
f_{l}\left(\Psi^{n}\right)=\frac{w_{l k}}{\sum_{j \neq l} w_{j k^{\prime}} \frac{\left|h_{l j k^{\prime}}^{n}\right|^{2}}{\sigma^{2}+\sum_{i \neq j} P_{i}^{n}\left|h_{i j k^{\prime}}^{n}\right|^{2}} \theta_{j l}^{n}}
$$

We have:

1. If $P_{l}^{n} \geq 0 \forall l, n$, then $f_{l}\left(\Psi^{n}\right)>0$.

2. If $P_{l}^{n} \geq P_{l}^{\prime n} \forall l, n$, then

$$
\frac{w_{l k}}{\sum_{j \neq l} \frac{w_{j j^{\prime}}\left|l_{l j k^{\prime}}^{n}\right|^{2} \theta_{j l}^{n}}{\sigma^{2}+\sum_{i \neq j} p_{i}^{n} \mid h_{i j k^{\prime}}^{n}}} \geq \frac{w_{l k}}{\sum_{j \neq l} \frac{w_{j k^{\prime}}\left|h_{l j k^{\prime}}^{n}\right|_{j j^{2}}^{n}}{\sigma^{2}+\sum_{i \neq j} P_{i}^{\prime}\left|h_{i j k^{\prime}}^{n}\right|^{2}}}
$$

Therefore, $f_{l}\left(\Psi^{n}\right) \geq f_{l}\left(\Psi^{\prime n}\right)$.

3. For $\rho>1$, we have:

$$
\begin{aligned}
\rho f_{l}\left(\Psi^{n}\right) & =\frac{w_{l k}}{\sum_{j \neq l} w_{j k^{\prime}} \frac{\left|k_{l j k^{\prime}}^{n}\right|^{2}+\left.\left.\sum_{i \neq j} \rho P_{i}^{n}\right|_{i j k^{\prime}} ^{n}\right|^{n}}{\sigma_{j l}^{n}}} \\
& >f_{l}\left(\rho \Psi^{\mathbf{n}}\right) .
\end{aligned}
$$

Thus, $f_{l}($.) satisfies the standard function properties. Based on Theorem 7 in [17], the power constrained function $\left[f_{l}(.)\right]_{0}^{S^{\max }}$ is also standard. Note that a fixed point of this function iteration must exist, since the original optimization problem has at least one local optimum. Together with Corollary 1 in [17], this implies that, starting from any initial $P_{l}^{n}(0), \theta$-IFEM- 1 is guaranteed to converge to a unique fixed point.

Further, based on Theorem 4 of [17], the asynchronous version of $\theta$-IFEM-1 defined by the following update 
equation, is guaranteed to converge to the same unique fixed point of $\theta$-IFEM-1:

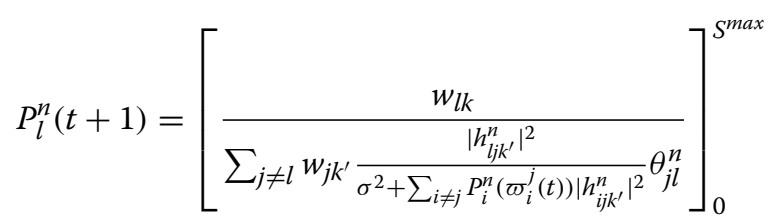

where the index $\varpi_{i}^{j}$ point to possibly outdated information of the $i$ th AN that is available at the processor of the jth AN.

Finally, note that High-SINR IFEM (HSIFEM) and $\theta$ IFEM-1 are based on different power update equations. Therefore, they can potentially converge to different unique fixed points.

\section{Connection with SCALE}

In [3], a power control algorithm named SCALE is proposed. The algorithm is motivated by geometric programming. SCALE is a two-stage algorithm, and in the notation of this paper can be thought of as having an inner loop to update the power $P_{l}^{n}$, and an outer loop to update the coefficients $\theta_{j l}^{n}$. The SCALE algorithm defines $\alpha_{j}^{n}=\frac{\operatorname{SINR}_{j}^{n}}{1+\operatorname{SINR}_{j}^{n}}$, so that $\theta_{j l}^{n}=\alpha_{j}^{n} / \alpha_{l}^{n}$. It runs iterative function evaluation with fixed $\alpha_{j}^{n}$ in the inner loop to update $P_{l}^{n}$, then updates $\alpha_{j}^{n}$ based on the resulting SINRs in the outer loop. IFEM1 proposed in this paper is essentially a simplification of SCALE. Instead of the two-stage process in which $\theta_{j l}^{n}$ is updated in an outer loop, IFEM-1 implicitly updates the power vector and $\theta_{j l}^{n}$ at the same time.

In addition, as shown in the simulation results presented later in this paper, the use of a single fixed $\theta_{j l}^{n}$ derived from the maximum transmit power level may already be near optimum (leading to $\theta$-IFEM- 1 ), thus $\theta_{j l}^{n}$ may not need to be updated at all. Further, at high SINR, $\theta_{j l}^{n}$ can be set to 1 , leading to HSIFEM. It is interesting to remark that although SCALE is motivated by geometric programming, the algorithm itself can be easily derived without geometric programming, as shown in this paper.

\section{Connection with iterative water-filling}

In [4], a power control algorithm named Modified Iterative Water-filling Algorithm (MIWFA) is proposed. MIWFA is again a two-stage algorithm. The inner loop iteratively finds the power level of every AN at every tone $n$ for fixed interference pricing terms $\tau_{j l}^{n}$ :

$$
P_{l}^{n}(t+1)=\left[\frac{w_{l k}}{\sum_{j \neq l} \tau_{j l}^{n}}-\frac{P_{l}^{n}(t)}{\operatorname{SINR}_{l}^{n}(t)}\right]_{0}^{S^{\max }}
$$

The outer loops then updates the pricing terms $\tau_{j l}^{n}$ :

$$
\tau_{j l}^{n}=w_{j k^{\prime}} \frac{\left|h_{l j k^{\prime}}^{n}\right|^{2}}{\sigma^{2}+\sum_{i \neq j} P_{i}^{n}\left|h_{i j k^{\prime}}^{n}\right|^{2}} \frac{\operatorname{SINR}_{j}^{n}}{1+\operatorname{SINR}_{j}^{n}}
$$

Iterations between the inner and outer loops are performed until convergence. IFEM-2, proposed in this paper, is essentially a simplification of MIWFA. Instead of the two-stage process in which the interference pricing terms $\tau_{j l}^{n}$ are updated in an outer loop, IFEM-2 implicitly updates the power vector and $\tau_{j l}^{n}$ at the same time.

\section{Comparison with Newton's method}

As a baseline comparison, we also describe the following Newton's method (NM) update equation as in [15]:

$$
P_{l}^{n}(t+1)=\left[P_{l}^{n}(t)+\mu \Delta P_{l}^{n}(t)\right]_{0}^{S^{\max }},
$$

where $\mu$ is the step size, and

$$
\Delta P_{l}^{n}(t)=\frac{\frac{w_{l k}}{P_{l}^{n}(t)}\left(1+\frac{1}{\operatorname{SINR}_{l}^{n}(t)}\right)^{-1}-\sum_{j \neq l} \tau_{j l}^{n}(t)}{\frac{w_{l k}}{\left(P_{l}^{n}(t)\right)^{2}}\left(1+\frac{1}{\operatorname{SINR}_{l}^{n}(t)}\right)^{-2}}
$$

where $\tau_{j l}^{n}$ is the interference price defined in (23).

The main disadvantage of the Newton's method is that the choice of step size cannot be easily done in a distributed fashion, and certainly not asynchronously. Note that the above Newton's update already omitted the offdiagonal terms of the Hessian matrix. To further simplify the computations required at each iteration of Newton's method, we also propose a high-SINR Newton's method (HSNM) where the update equation (25) is approximated as

$$
\Delta P_{l}^{n}(t)=\frac{\frac{w_{l k}}{P_{l}^{n}(t)}-\sum_{j \neq l} w_{j k^{\prime}} \frac{\left|h_{l j k^{\prime}}^{n}\right|^{2}}{\sigma^{2}+\sum_{i \neq j} P_{i}^{n}(t)\left|h_{i j k^{\prime}}^{n}\right|^{2}}}{\frac{w_{l k}}{\left(P_{l}^{n}(t)\right)^{2}}}
$$

\section{Distributed implementation}

The above methods IFEM-1, IFEM-2, $\theta$-IFEM-1, $\theta$-IFEM2, HSIFEM, NM, and HSNM can be put together under a generic power update equation:

$$
P_{l}^{n}(t+1)=\vartheta\left(\tau_{j l}^{n}(t), P_{l}^{n}(t), \operatorname{SINR}_{l}^{n}(t)\right)
$$

Typically, $P_{l}^{n}$ is known and $\operatorname{SINR}_{l}^{n}$ can be easily measured at each AN. A distributed implementation of power allocation (27) is, therefore, possible if ANs exchange the variables $\tau_{j l}^{n}$. As mentioned earlier, $\tau_{j l}^{n}$ has the interpretation of being the interference price. This exchange of information amounts to reasonable overhead for distributed implementation. Note that $\theta$-IFEM- 1 and HSIFEM can, in addition, be implemented asynchronously at each AN using possibly outdated power information, as shown in Proposition 1. 
Methods based on average channel gains

All the methods above are based on a per-tone channel measurements. Those measurements are typically provided to either a central server for further centralized processing, or to each of the several access nodes for distributed processing. In practice, to minimize the overhead of such exchange of information, the measurements can alternatively provide the frequency domain average channel gains. The proposed methods above can make use of the average channel gains instead. In the following, we list these "average gain" methods, and subsequently quantify their performance in a typical wireless backhaul network. These methods are of particular interest when the delay spread of the wireless propagation environment is limited.

Define the average channel gain $\left|\bar{h}_{j l k}\right|^{2}$ as:

$$
\left|\bar{h}_{j l k}\right|^{2}=\frac{1}{N} \sum_{n=1}^{N}\left|h_{j l k}^{n}\right|^{2}, \quad \forall(j, l, k)
$$

By substituting the average gains in the equations of the methods presented earlier, the update equations of the resulting methods can be summarized as follows:

- Average Gain IFEM-1 (AG IFEM-1):

$$
P_{l}^{n}(t+1)=\left[\frac{w_{l k} \frac{\overline{\operatorname{SINR}}_{l}^{n}(t)}{1+\overline{\operatorname{SINR}}_{l}^{n}(t)}}{\sum_{j \neq l} \bar{\tau}_{j l}^{n}(t)}\right]_{0}^{S^{\max }}
$$

where

$$
\bar{\tau}_{j l}^{n}(t)=\frac{w_{j k^{\prime}}\left|\bar{h}_{l j k^{\prime}}\right|^{2}}{\sigma^{2}+\sum_{i \neq j} P_{i}^{n}(t)\left|\bar{h}_{i j k^{\prime}}\right|^{2}} \frac{{\overline{\operatorname{SINR}_{j}}}^{2}(t)}{1+\overline{\operatorname{SINR}}_{j}^{n}(t)}
$$

and

$$
\overline{\operatorname{SINR}}_{j}^{n}(t)=\frac{P_{j}^{n}(t)\left|\bar{h}_{j j k^{\prime}}\right|^{2}}{\Gamma\left(\sigma^{2}+\sum_{i \neq j} P_{i}^{n}(t)\left|\bar{h}_{i j k^{\prime}}\right|^{2}\right)}
$$

- Average Gain IFEM-2 (AG IFEM-2):

$$
P_{l}^{n}(t+1)=\left[\frac{w_{l k}}{\sum_{j \neq l} \bar{\tau}_{j l}^{n}(t)}-\frac{P_{l}^{n}(t)}{\operatorname{SINR}_{l}^{n}(t)}\right]_{0}^{S^{\max }}
$$

Average Gain $\theta$-IFEM-1 (AG $\theta$-IFEM-1):

$$
P_{l}^{n}(t+1)=\left[\frac{w_{l k}}{\sum_{j \neq l} w_{j k^{\prime}} \frac{\left|\bar{h}_{l j k^{\prime}}\right|^{2}}{\sigma^{2}+\sum_{i \neq j} P_{i}^{n}(t)\left|\bar{h}_{i j k^{\prime}}^{n}\right|} \bar{\theta}_{j l}^{n}}\right]_{0}^{S^{\max }}
$$

where

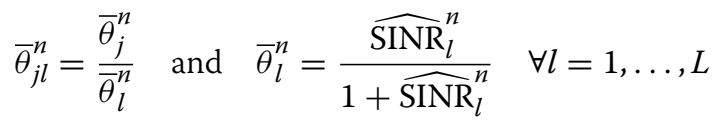

and

$$
\widehat{\operatorname{SINR}}_{l}^{n}=\frac{S_{\text {max }}\left|\bar{h}_{l l k}\right|^{2}}{\Gamma\left(\sigma^{2}+\sum_{i \neq j} S_{\text {max }}\left|\bar{h}_{j l k}\right|^{2}\right)}
$$

- Average Gain $\theta$-IFEM-2 (AG $\theta$-IFEM-2):

$$
P_{l}^{n}(t+1)=\left[\frac{w_{l k}}{\sum_{j \neq l} \frac{w_{j k^{\prime}}\left|\bar{h}_{l j k^{\prime}}\right|^{2}}{\sigma^{2}+\sum_{i \neq j} P_{i}^{n}(t)\left|\bar{h}_{i j k^{\prime}}\right|^{2}} \bar{\theta}_{j}^{n}}-\frac{P_{l}^{n}(t)}{\frac{\bar{\theta}_{l}^{n}}{1-\bar{\theta}_{l}^{n}}}\right]_{0}^{S^{\max }}
$$

- Average Gain HSIFEM (AG HSIFEM):

$$
P_{l}^{n}(t+1)=\left[\frac{w_{l k}}{\sum_{j \neq l} w_{j k^{\prime}} \frac{\left|\bar{h}_{l j k^{\prime}}\right|^{2}}{\sigma^{2}+\sum_{i \neq j} P_{i}^{n}(t)\left|\bar{h}_{i j k^{\prime}}\right|^{2}}}\right]_{0}^{S^{\max }}
$$

Average Gain Newton's method (AG NM)

$$
P_{l}^{n}(t+1)=\left[P_{l}^{n}(t)+\mu \Delta P_{l}^{n}(t)\right]_{0}^{S^{\max }},
$$

where $\mu$ is the step size, and

$$
\Delta P_{l}^{n}(t)=\frac{\frac{w_{l k}}{P_{l}^{n}(t)}\left(1+\frac{1}{\overline{\operatorname{SINR}}_{l}^{n}(t)}\right)^{-1}-\sum_{j \neq l} \bar{\tau}_{j l}^{n}(t)}{\frac{w_{l k}}{\left(P_{l}^{n}(t)\right)^{2}}\left(1+\frac{1}{\overline{\operatorname{SINR}}_{l}^{n}(t)}\right)^{-2}}
$$

- Average Gain HSNM (AG HSNM):

$$
P_{l}^{n}(t+1)=\left[P_{l}^{n}(t)+\mu \Delta P_{l}^{n}(t)\right]_{0}^{S^{\max }},
$$

where

$$
\Delta P_{l}^{n}(t)=\frac{\frac{w_{l k}}{P_{l}^{n}(t)}-\sum_{j \neq l} w_{j k^{\prime}} \frac{\left|\bar{h}_{l j k^{\prime}}\right|^{2}}{\sigma^{2}+\sum_{i \neq j} P_{i}^{n}(t)\left|\bar{h}_{i j k^{\prime}}\right|^{2}}}{\frac{w_{l k}}{\left(P_{l}^{n}(t)\right)^{2}}}
$$

Finally, there are situations in which the channel measurement can be done on a per-tone basis, but the physical transmission platforms only permit each AN to allocate one value of power across all frequency tones. In this case, we take the average power (AP) values of IFEM algorithms, and call the resulting method AP algorithms. For example, AP HSIFEM takes the average power value of the HSIFEM. 
Table 1 System model parameters

\begin{tabular}{lc}
\hline Cellular layout & Hexagonal \\
\hline Number of ANs & 7 \\
\hline Frequency Reuse & 1 \\
\hline Number of RTs per AN & 4 \\
\hline AN-to-AN Distance & $d_{1}$ \\
\hline AN-to-RT Distance & $d_{2}$ \\
\hline Duplex & TDD \\
\hline Channel Bandwidth & $10 \mathrm{MHz}$ \\
\hline AN Max Tx Power per Subcarrier & $-32.70 \mathrm{dBw}$ \\
\hline SINR Gap & $12 \mathrm{~dB}$ \\
\hline Total Noise Power Per Subcarrier & $-158.61 \mathrm{dBw}$ \\
\hline BS Tx Antenna No. & 1 \\
\hline MS Rx Antenna No. & 1 \\
\hline Sampling Frequency & $11.2 \mathrm{MHz}$ \\
\hline FFT Size & 1024 \\
\hline Distance-dependent Path Loss & $128.1+37.6$ log $10(d)$ \\
\hline Multipath Delay Profile & SUl-3 Terrain type B \\
\hline
\end{tabular}

In practice, the AP algorithms require an additional module which runs the algorithms on a per-tone basis to find the set of powers $P_{l}^{n}$, and then returns an average value of the power $P_{l}$ for each $\mathrm{AN} l$, i.e. $P_{l}=\sum_{n=1}^{N} P_{l}^{n}$. This approach for solving the power optimization subject to constant power across tones is obviously suboptimal; nevertheless, this proposed solution always outperforms
Table 2 Methods behavior over 7 ANs, 4 RTs per AN

\begin{tabular}{lcc}
\hline Sum rate in bps/Hz & $\boldsymbol{d}_{\mathbf{1}} \mathbf{0} \mathbf{0 . 5} \mathbf{k m}$ & $\boldsymbol{d}_{\mathbf{1}} \mathbf{1} \mathbf{k m}$ \\
\hline SIFEM & 60.68 & 91.33 \\
\hline AG HSIFEM & 60.67 & 91.33 \\
\hline HSNM & 60.68 & 91.33 \\
\hline AG HSNM & 60.67 & 91.33 \\
\hline IFEM-1 & 62.61 & 91.58 \\
\hline AG IFEM-1 & 62.59 & 91.57 \\
\hline IFEM-2 & 62.61 & 91.58 \\
\hline AG IFEM-2 & 62.59 & 91.57 \\
\hline NM & 62.61 & 91.58 \\
\hline AG NM & 62.59 & 91.57 \\
\hline AP HSIFEM & 56.88 & 88.13 \\
\hline Max Power Method & 53.01 & 86.22 \\
\hline IFEM-1 Gain & $18.1 \%$ & $6.2 \%$ \\
\hline
\end{tabular}

$d_{1}$ is the AN-to-AN distance. AN-to-RT distance is $150 \mathrm{~m}$.

conventional systems with maximum power transmission, as the simulations suggest.

\section{Simulations}

This section evaluates the benefit of the proposed power spectrum optimization methods and verifies their convergence behavior in the downlink of a wireless backhaul network comprising seven ANs, and 4 RTs per AN, over $10 \mathrm{MHz}$ bandwidth. The transmission of each AN to its own RTs interferes with the other ANs' transmissions. RTs belonging to one AN are separated from each other

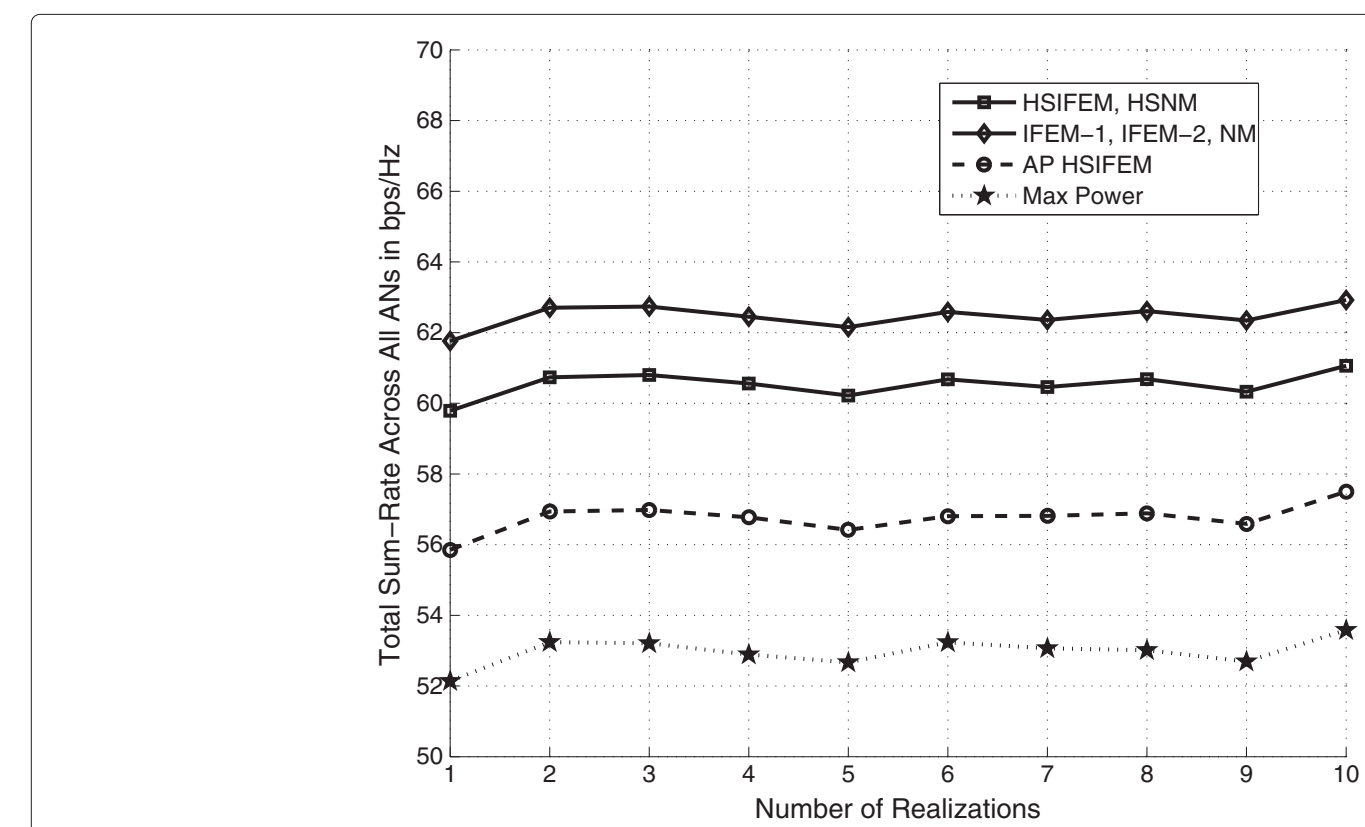

Figure 2 Sum-rate in bps/Hz over 7 ANs, 4 RTs per AN. AN-to-AN distance is $0.5 \mathrm{~km}$. AN-to-RT distance is $150 \mathrm{~m}$. 
Table 3 Methods behavior over 7 ANs, 4 RTs per AN

\begin{tabular}{lcc}
\hline Sum rate in bps/Hz & $\begin{array}{c}\text { Cell-edge } \\
\left(\boldsymbol{d}_{\mathbf{2}} \mathbf{3} \mathbf{3 3 \mathbf { m }}\right)\end{array}$ & $\begin{array}{c}\text { Cell-center } \\
\left(\boldsymbol{d}_{\mathbf{2}} \mathbf{=} \mathbf{1 2 5} \boldsymbol{\mathbf { m }}\right)\end{array}$ \\
\hline HSIFEM & 34.84 & 78.39 \\
\hline AG HSIFEM & 34.83 & 78.38 \\
\hline HSNM & 34.84 & 78.39 \\
\hline AG HSNM & 34.83 & 78.38 \\
\hline IFEM-1 & 41.11 & 78.77 \\
\hline AG IFEM-1 & 41.09 & 78.76 \\
\hline IFEM-2 & 41.11 & 78.77 \\
\hline AG IFEM-2 & 41.09 & 78.76 \\
\hline NM & 41.11 & 78.77 \\
\hline AG NM & 41.09 & 78.76 \\
\hline AP HSIFEM & 31.54 & 75.00 \\
\hline Max Power Method & 30.54 & 71.91 \\
\hline IFEM-1 Gain & $34.6 \%$ & $9.5 \%$ \\
\hline AN-to-AN distance is 0.5km & &
\end{tabular}

$\mathrm{AN}$-to-AN distance is $0.5 \mathrm{~km}$.

using OFDMA with 1024 subcarriers, where only one RT is active at each frequency tone. The parameters used in simulation are as outlined in Table 1. The AN-toAN distance is set to $d_{1}$; the AN-to-RT distance is set to $d_{2}$. Both $d_{1}$ and $d_{2}$ vary so as to study the performance of the proposed methods for various topologies. For illustration purposes, the weighting factors $w_{l k}$ in problem (4) are set to $1, \forall(l, k)$, which allows a sum-rate comparison.
Figure 2 shows the sum-rate performance over all ANs for a network with AN-to-AN distance $d_{1}=0.5 \mathrm{~km}$ and AN-to-RT distance $d_{2}=0.15 \mathrm{~km}$ over different realizations of the channel. First, despite the difficulty in proving the convergence of full IFEM algorithms, both IFEM-1 and IFEM-2 are always observed to converge. In addition, they have similar performance to each other, and have similar performance to the Newton's method. Figure 2 also shows that there is a small performance loss due to the high SINR approximation (i.e. HSIFEM and HSNM have a lower performance as compared to IFEM-1, IFEM-2, and NM). Nevertheless, HSIFEM and HSNM outperform the maximum power method significantly.

We also observe that AP HSIFEM, which allocates equal power in each $\mathrm{AN}$ across all the tones, is always superior to the maximum power method. However, there is some performance loss as compared to HSIFEM/HSNM where per-tone adaptation is enabled.

Tables 2 and 3 illustrate the performance of the proposed methods for different network topologies. Table 2 considers the effects of cell sizes, and shows that the benefit of power optimization is more pronounced in a small-cell setting, where the interference level is higher. Table 3 examines the effect of RT locations within each cell. It is shown that the benefit of power optimization is noticeably higher for cell-edge users, where the interference is larger.

It can be observed from Tables 2 and 3 that HSIFEM and HSNM always have the same performance, as both employ a high-SINR approximation. At the cell-edge of

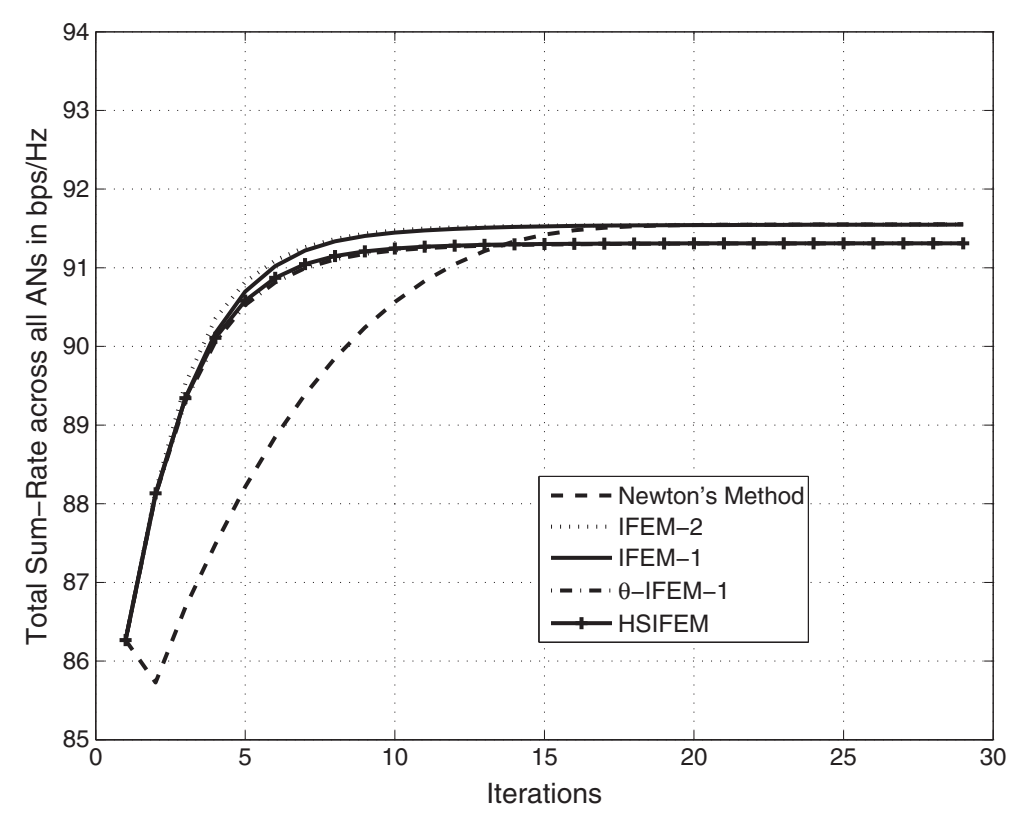

Figure 3 Sum-rate in bps/Hz versus the number of iterations, over 7 ANs, 4 RTs per AN. AN-to-AN distance is $1 \mathrm{~km}$. AN-to-RT distance is $150 \mathrm{~m}$. It shows the convergence of the different methods at high SINR. 


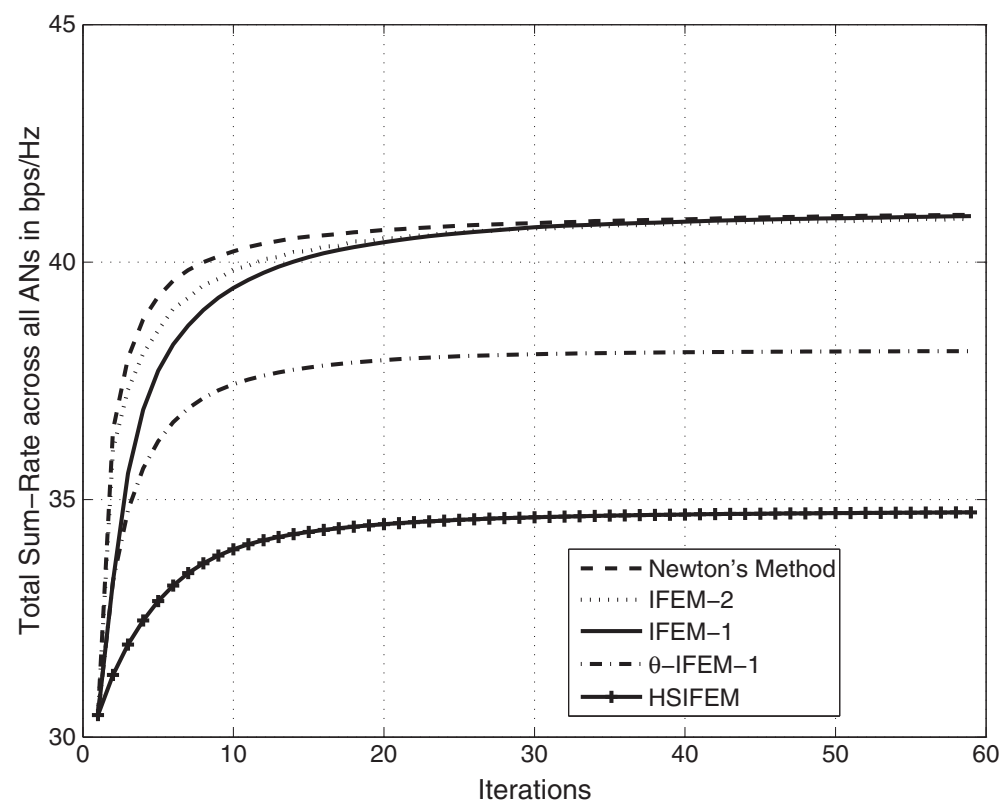

Figure 4 Sum-rate in bps/Hz versus the number of iterations, over $\mathbf{7 ~ A N s , ~} 4$ RTs per AN. AN-to-AN distance is $0.5 \mathrm{~km}$. AN-to-RT distance is $333 \mathrm{~m}$. It shows the convergence of the different methods at low SINR.

small cells, where the SINR level is not sufficiently large to justify the high-SINR approximation, HSIFEM and HSNM become inferior to IFEM-1, IFEM-2 and NM. This is, however, not the case for cell-center users, e.g. the case of $d_{1}=1 \mathrm{~km}, d_{2}=150 \mathrm{~m}$ as shown in Table 2 and the case of $d_{1}=0.5 \mathrm{~km}, d_{2}=125 \mathrm{~m}$ as shown in Table 3 , where SINR values are larger, and where HSIFEM, HSNM, IFEM-1,IFEM-2 and NM all have similar performance. In all cases, IFEMs always remain superior to the maximum power method.

Tables 2 and 3 also show that the methods that rely on the frequency domain average channel gain have a very

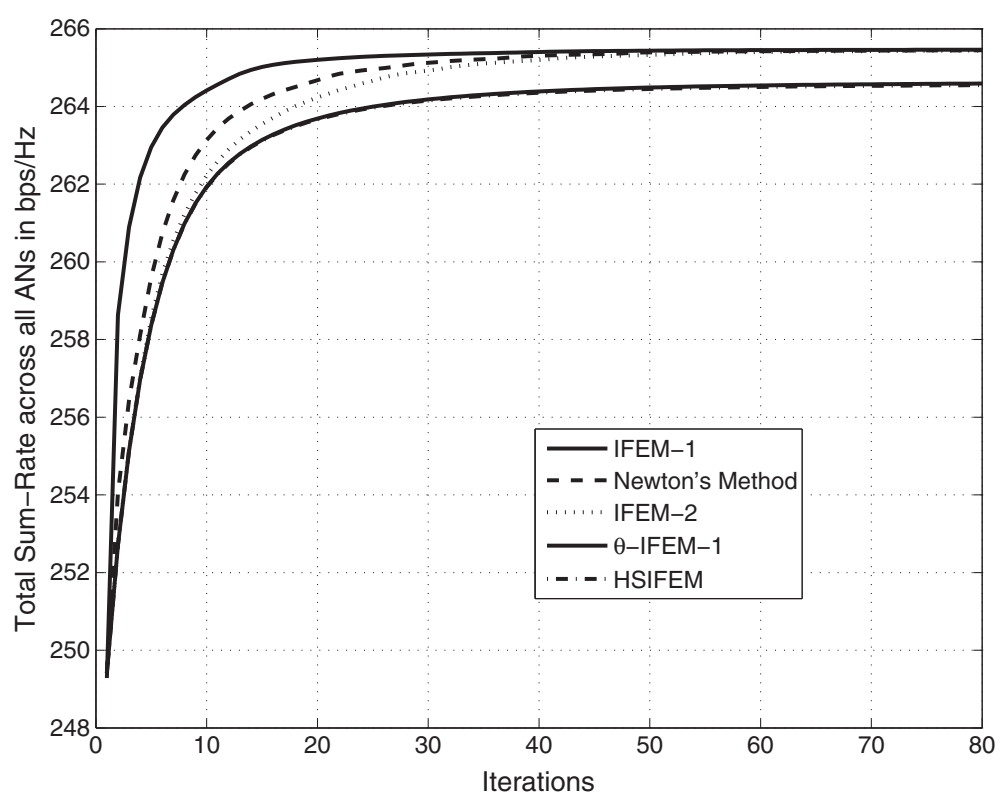

Figure 5 Sum-rate in bps/Hz versus the number of iterations, over 21 ANs, 4 RTs per AN. AN-to-AN distance is $1 \mathrm{~km}$. AN-to-RT distance is $150 \mathrm{~m}$. It shows the convergence of the different methods in a large network at high SINR. 


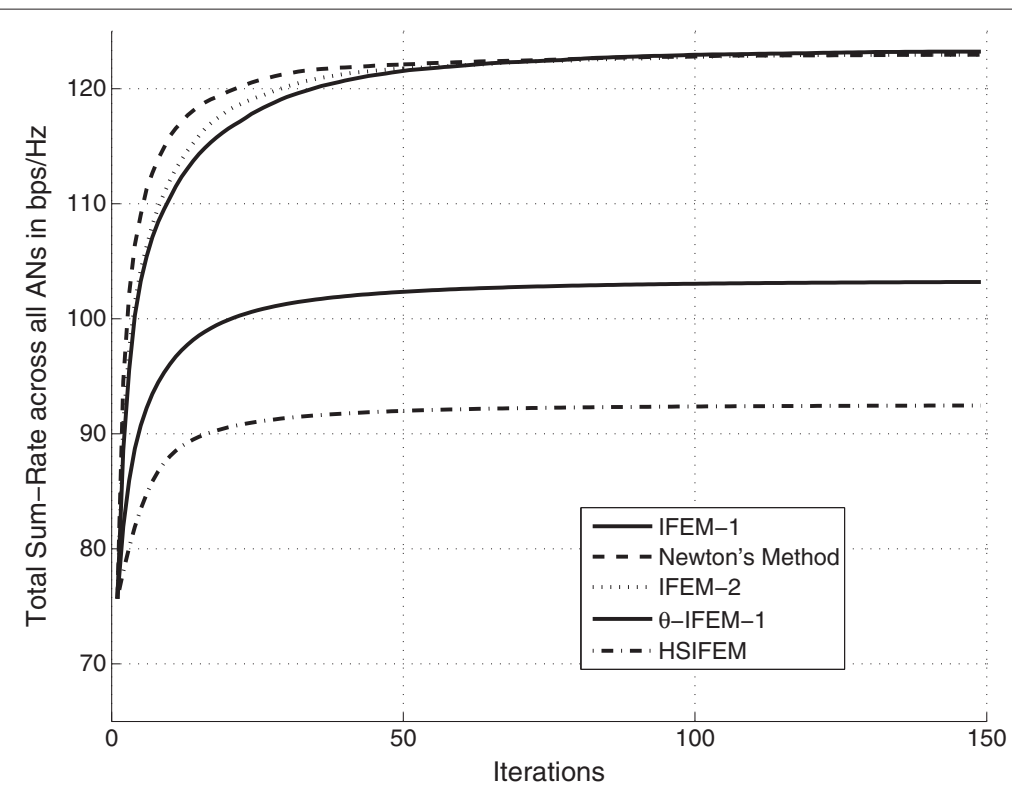

Figure 6 Sum-rate in bps/Hz versus the number of iterations, over 21 ANs, 4 RTs per AN. AN-to-AN distance is $0.5 \mathrm{~km}$. AN-to-RT distance is $333 \mathrm{~m}$. It shows the convergence of the different methods in a large network at low SINR.

similar performance to the methods that are based on the per-tone channel gain measurements. This is due to the fact that a typical fixed wireless backhaul channel is relatively flat fading. Our simulation assumes an SUI-3 model with Terrain type $B$, in which the delay profile has three taps; the k-factors of the three taps are 3,0 , and 0 ; the relative taps delays are $0,0.4$, and 0.9 in microseconds.
Figures 3 and 4 compare the convergence performance of IFEM algorithms with the Newton's method. For fair comparison, the Newton's method is plotted here with a constant step size of 1 . As seen in Figure 3, because of the constant step size, the Newton's method has a poor performance initially, and IFEM converges faster overall. Note that the convergence speed comparison depends

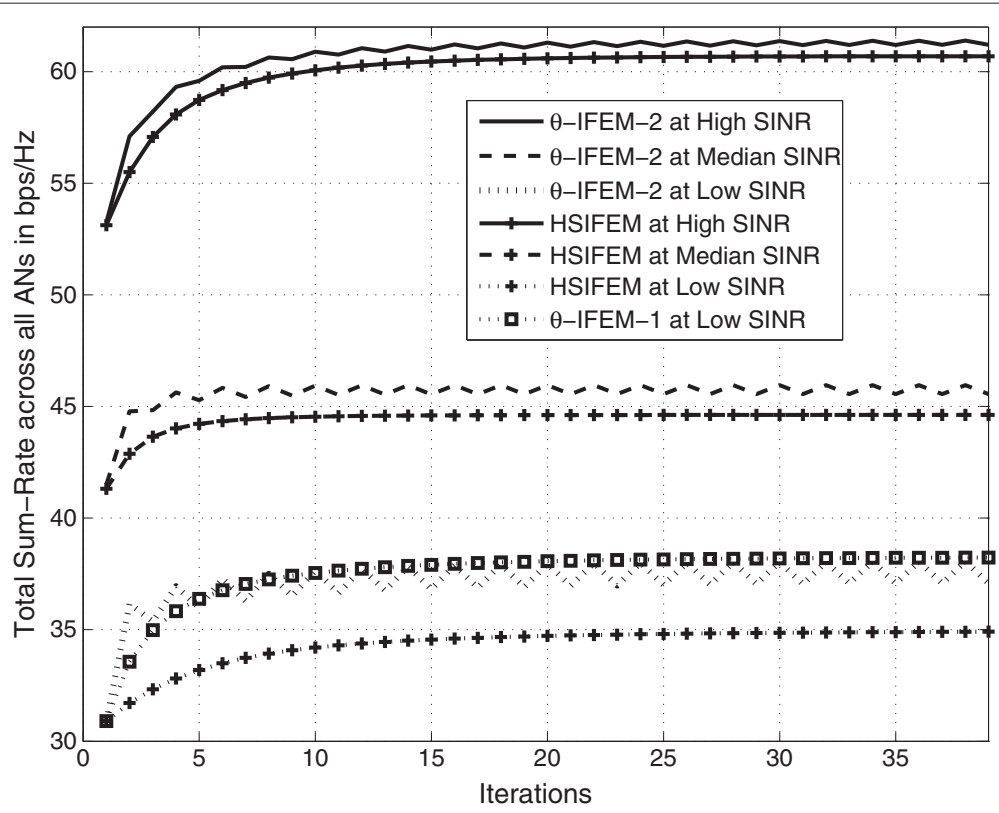

Figure 7 Sum-rate in bps/Hz versus the number of iterations, over 7 ANs, 4 RTs per AN, for different SINR levels. It shows the oscillatory convergence behavior of $\theta$-IFEM- 2 . 
on the SINR. Figure 3 corresponds to a high SINR situation, where IFEM outperforms the Newton's method. Figure 4 shows an opposite situation, at a relatively low SINR, where the convergence of the Newton's method is faster than IFEM-1 and IFEM-2. Note that at high SINR, the achievable sum-rate performances of HSIFEM, $\theta$-IFEM-1, IFEM-1, IFEM-2 and Newton's method are similar, while at low SINR, IFEM-1, IFEM-2 and Newton's method outperform $\theta$-IFEM-1, which in term outperforms HSIFEM.

To study the convergence behavior of the proposed methods in a larger network, we simulate a wireless network comprising 21 ANs, and 4 RTs per AN. We plot the total sum-rate versus the number of iterations as shown in Figures 5 and 6. Figure 5 corresponds to a high SINR situation where IFEM-1 outperforms the other methods. Figure 6 corresponds to a low SINR situation, where Newton's method shows the best convergence behavior. IFEM-1, IFEM-2 and Newton's method all outperform $\theta$ IFEM-1, which in term outperforms HSIFEM. We note that $\theta$-IFEM-1 and HSIFEM, however, are simpler to implement and already show respective gains of $35 \%$ and $21 \%$ as compared to maximum power transmission strategy.

Finally, to illustrate the convergence behavior of $\theta$ IFEM-2, we simulate a 7-AN network, with 4 RTs per AN. We plot the total sum-rate versus the number of iterations as shown in Figure 7. Unlike the other proposed methods in the paper, $\theta$-IFEM-2 shows an oscillatory convergence behavior, especially at low SINR. At large SINR level, however, Figure 7 shows how $\theta$-IFEM- 2 convergence behavior resembles that of a standard function, i.e. HSIFEM in this case, which is expected, since HSIFEM is derived under large SINR assumption. We finally note that not only $\theta$-IFEM1 is guaranteed to converge always, but also its performance is superior to $\theta$-IFEM-2, as shown in the figure.

\section{Conclusion}

Given the scarcity of the available radio resources, the performance of future wireless networks would be expected to depend on the feasibility of the dynamic power spectrum optimization methods. This paper presents a class of very simple yet effective power control methods to manage interference in wireless systems. The proposed methods represent efficient ways of updating the power spectral density levels for all transmitters, based on either the frequency domain channel gains, or the frequency domain channel average gains. The proposed methods are based on the observation that different manipulations of the gradient of the objective function give different power update equations. These methods have low computational complexity and fast convergence. Their performance is similar to the full-blown Newton's method, but without the need for step size choices. They can also be implemented in a distributed fashion and asynchronously at each transmitter, and are therefore excellent fits for practical applicability.

\section{Competing interests}

The material in this paper has been filed as a United States patent under the title "Interference mitigation with scheduling and dynamic power spectrum allocation for wireless networks" by BLiNQ Networks Inc. Hayssam Dahrouj and Taiwen Tang have been employed by BLiNQ Networks Inc.

\section{Acknowledgements}

The authors acknowledge Natural Science and Engineering Research Council (NSERC) ENGAGE program and BLiNQ Networks Inc. for funding this work. The authors also wish to thank Mr. Steve Beaudin who was supportive and helpful during the project. This paper has been presented in part at IEEE Globecom First Workshop on Distributed Antenna Systems for Broadband Mobile

Communications, Houston, TX, USA, Dec 2011 [18], and in part at 26th Biennial Symposium on Communications, Kingston, Ontario, Canada, May 2012 [19].

Received: 13 February 2012 Accepted: 26 June 2012

Published: 6 August 2012

\section{References}

1. R Cendrillon, W Yu, M Moonen, J Verlinden, T Bostoen, Optimal multiuser spectrum balancing for digital subscriber lines. IEEE Trans. Commun. 54(5), 922-933 (2006)

2. WYu, R Lui, Dual methods for nonconvex spectrum optimization of multicarrier systems. IEEE Trans. Commun. 54(6), 1310-1322 (2006)

3. J Papandriopoulos, JS Evans, Low-complexity distributed algorithms for spectrum balancing in multi-user DSL networks. in Proc. IEEE Inter. Conf. Commun. (ICC). (Turkey: Istambul, 2006

4. W Yu, Multiuser water-filling in the presence of crosstalk. in Inform. Theory and Appl. Workshop. San Diego: U.S.A., 2007

5. P Tsiaflakis, M Diehl, M Moonen, Distributed spectrum management algorithms for multi-user DSL networks. IEEE Trans. Signal Process. 56(10), 4825-4843 (2008)

6. J Huang, RA Berry, ML Honig, Distributed interference compensation for wireless networks. IEEE J. Select. Areas Commun. 24(5), 1074-1084 (2006)

7. J Yuan, W Yu, Distributed cross-layer optimization of wireless sensor networks: A game theoretic approach. in Proc. IEEE Global Telecommun. Conf. (Globecom). U.S.A.: San Francisco, 2006

8. C Shi, RA Berry, ML Honig, Distributed interference pricing for OFDM wireless networks with non-separable utilities. Princeton: NJ, 2008)

9. F Wang, M Krunz, S Cui, Price-based spectrum management in cognitive radio networks. IEEE J. Sel. Top. Signal Processing. 1(2), 74-87 (2008)

10. J Huang, VG Subramanian, R Agrawal, R Berry, Downlink scheduling and resource allocation for OFDM systems. in Proc. Conf. on Inform. Sciences and Systems (CISS). (Princeton: NJ, 1271-1279, 2006)

11. J Huang, VG Subramanian, R Agrawal, R Berry, Joint scheduling and resource allocation in uplink OFDM systems for broadband wireless access networks. IEEE J. Sel. Top. Signal Process. 27(2), 226-234 (2009)

12. SG Kiani, D Gesbert, Optimal and distributed scheduling for multicell capacity maximization. IEEE Trans. Wireless Commun. 7, 288-297 (2008)

13. L Venturino, N Prasad, X Wang, Coordinated Scheduling and Power Allocation in Downlink Multicell OFDMA Networks. IEEE Trans. Veh. Technol. 58(6), 2835-2848 (2009)

14. AL Stolyar, H Viswanathan, Self-organizing dynamic fractional frequency reuse for best-effort traffic through distributed inter-cell coordination. in Proc. INFOCOM, 1287-1295, (2009)

15. W Yu, T Kwon, C Shin, Joint Scheduling and Dynamic Power Spectrum Optimization for Wireless Multicell Networks. in Conference on Information Science and Systems (CISS). (Princeton: NJ, 2010

16. LP Qian, Y Jun, J Huang, MAPEL: Achieving Global Optimality for a Non-Convex Wireless Power Control Problem. IEEE Trans. Wireless Commun. 8(3), 1553-1563 (2009)

17. R Yates, A framework for uplink power control in cellular radio systems. IEEE J. Sel. Areas Commun. 13(7), 1341-1347 (1995) 
18. H Dahrouj, W Yu, T Tang, S Beaudin, Power Spectrum Optimization for Interference Mitigation Via Iterative Function Evaluation. in Proc. First Workshop on Distributed Antenna Systems for Broadband Mobile Communications IEEE Global Telecommun. Conf. (Globecom). Houston: U.S.A., 2011

19. H Dahrouj, W Yu, T Tang, On Power Spectrum Optimization by Iterative Function Evaluation. in Proc. 26th Biennial Symposium on Communications. Canada: Kingston, Ontario, 2012

doi:10.1186/1687-1499-2012-244

Cite this article as: Dahrouj et al:: Power spectrum optimization for interference mitigation via iterative function evaluation. EURASIP Journal on Wireless Communications and Networking 2012 2012:244.

Submit your manuscript to a SpringerOpen ${ }^{\mathcal{O}}$ journal and benefit from:

- Convenient online submission

- Rigorous peer review

- Immediate publication on acceptance

- Open access: articles freely available online

- High visibility within the field

- Retaining the copyright to your article

Submit your next manuscript at springeropen.com 